\title{
Giant Cell Arteritis: A Systematic Review of the Qualitative and Semiquantitative Methods to Assess Vasculitis with 18F-Fluorodeoxyglucose Positron Emission Tomography
}

\author{
Cristina Puppo, ${ }^{1}$ Michela Massollo, ${ }^{2}$ Francesco Paparo, ${ }^{1}$ Dario Camellino, ${ }^{3}$ \\ Arnoldo Piccardo, ${ }^{2}$ Mehrdad Shoushtari Zadeh Naseri, ${ }^{2}$ Giampiero Villavecchia, ${ }^{2}$ \\ Gian Andrea Rollandi, ${ }^{1}$ and Marco Amedeo Cimmino ${ }^{3}$ \\ ${ }^{1}$ Department of Radiology, Department of Diagnostic Imaging, E.O. Ospedali Galliera, Mura della Cappuccine 14, 16128 Genoa, Italy \\ ${ }^{2}$ Nuclear Medicine Unit, Department of Diagnostic Imaging, E.O. Ospedali Galliera, Mura della Cappuccine 14, 16128 Genoa, Italy \\ ${ }^{3}$ Research Laboratory and Academic Unit of Clinical Rheumatology, Department of Internal Medicine, University of Genoa, \\ V.le Benedetto XV 6, 16132 Genoa, Italy \\ Correspondence should be addressed to Michela Massollo; michela.massollo@galliera.it
}

Received 5 June 2014; Accepted 28 July 2014; Published 1 September 2014

Academic Editor: Alberto Signore

Copyright (C) 2014 Cristina Puppo et al. This is an open access article distributed under the Creative Commons Attribution License, which permits unrestricted use, distribution, and reproduction in any medium, provided the original work is properly cited.

\begin{abstract}
Giant cell arteritis (GCA) is the most common vasculitis affecting medium and large vessels. It shows a close clinical association with polymyalgia rheumatica (PMR), a musculoskeletal inflammatory disorder, which is clinically characterized by girdles pain and stiffness. 18F-Fluorodeoxyglucose (18F-FDG) positron emission tomography (PET) is an effective tool for the diagnosis, grading, and follow-up of patients affected by GCA involving the aorta and its proximal branches, but the lack of a standardized method for the assessment of vascular inflammation remains a critical issue, potentially leading to misclassification. In our systematic review, including 19 original articles for a total of 442 GCA patients (with or without PMR symptoms) and 535 healthy controls, we described the different qualitative, semiquantitative and combined methods that have been proposed throughout the literature for assessing the presence and grading the severity of GCA-related vascular inflammation on 18F-FDG PET scans, focusing on the diagnostic performance and examining their respective advantages and limitations. The majority of the included studies adopted qualitative methods of PET image analysis, which are less sensitive but more specific than semiquantitative ones. Among the semiquantitative approaches, the aortic-to-blood pool uptake ratio of the aortic arch seems to be the most accurate method.
\end{abstract}

\section{Introduction}

Giant cell arteritis (GCA) is the most common vasculitis affecting medium and large vessels, with an incidence of 7-18 cases per 100,000 individuals and with women affected twice as often as men $[1,2]$. GCA was initially described as temporal arteritis (Horton disease), but about $15-27 \%$ of patients have extracranial involvement, since the entire aorta and all its branches can be affected, including the carotid, subclavian, and iliac arteries [3-5]. Polymyalgia rheumatica (PMR) is an inflammatory disorder, two to three times more common than GCA and clinically characterized by girdles pain and stiffness. PMR can occur before and simultaneously with or develop after clinical manifestations of GCA [6-10].
Population-based studies have shown that PMR occurs in about $50 \%$ of patients with GCA, and approximately $15 \%$ $30 \%$ of PMR patients develop GCA $[1,11]$. The presence of different clinical features common to both PMR and GCA (e.g., older age at onset with progressively increasing incidence rates after 50 years, similar sex ratio, substantial increase of acute-phase reactants, and rapid responsiveness to glucocorticoids) has suggested that they might be different manifestations of the same underlying process [1].

Although etiology, development mechanisms, and targets of inflammatory damage of both GCA and PMR have not been yet defined, there is increasing evidence that a combination of genetic, immunogenetic [12-17], and environmental factors may play a pivotal role $[12,18,19]$. 
Early detection of the involvement of thoracic aorta and its branches plays a fundamental role in patient management and treatment. Thoracic aortic aneurysms are more frequent in patients with GCA than in nonaffected people and tend to arise several years after the diagnosis, when other symptoms are less evident $[5,20]$.

Over the past recent years, 18F-FDG PET, computed tomography (CT) angiography, and magnetic resonance imaging (MRI) have revealed that extracranial involvement in GCA is more frequent than previously anticipated, occurring in $30-74 \%$ of patients [21-24].

$18 \mathrm{~F}-\mathrm{FDG}$ PET is a functional imaging technique that has become an established tool in oncology [5] but it has demonstrated also a promising role in the field of inflammatory diseases $[4,5]$. The main limitation of 18 F-FDG PET/CT to become a reliable diagnostic tool is the lack of a standardized definition of vascular inflammation based on the intensity of the glucose analogue uptake. Several authors have proposed various $18 \mathrm{~F}-\mathrm{FDG}$ PET diagnostic criteria.

This systematic review is focused on the different qualitative and semiquantitative methods for diagnosis and grading of vascular inflammation in GCA patients (with or without associated PMR) by means of 18F-FDG PET. We also assessed the diagnostic performance and the clinical value of each method of evaluation.

Takayasu arteritis (TA) was not included in our analysis because, though sharing apparent similar FDG distributions with GCA, its target population, pathophysiology, evolution, and prognosis are not comparable with those of GCA.

\section{Materials and Methods}

2.1. Database Search. A systematic literature research was performed up to April 2014, with no time limits. PubMed and the Cochrane Library were searched for articles written in English that addressed the issue of 18F-FDG PET as a diagnostic tool in GCA with or without associated PMR. We used the MeSH query "giant cells arteritis" or "polymyalgia rheumatica" and "positron emission tomography."

A first selection was based on the exclusion of review articles, meta-analyses, abstracts, editorials or letters, case reports, and studies investigating 3 or fewer patients because they failed to provide sufficient evidence-based data. In this first stage, two researchers independently reviewed titles and abstracts of all retrieved articles. Studies addressing $18 \mathrm{~F}-$ FDG-PET as diagnostic tool in GCA/PMR were included, while articles related to other vasculitides were excluded.

At the second stage, the same two researchers independently assessed the full-text version of all articles that were found to be potentially eligible for inclusion, using the same inclusion and exclusion criteria as mentioned above (Figure 1). At both stages, disagreements between the two researchers were discussed and resolved by consensus.

\section{Results}

A total of 199 citations were found using the database search. Nineteen full-text articles, written from 1999 to 2014, were included. Of them, 13 were prospective and 6 retrospective studies. In order to assess vascular inflammation in GCA, with or without associated PMR, 10 studies used exclusively qualitative 18F-FDG uptake criteria, 6 used only semiquantitative criteria, and 3 adopted both qualitative and semiquantitative criteria (Table 1).

3.1. Qualitative Methods for the Assessment of 18F-FDG PET. Ten studies [25-28, 30-32, 36, 38, 39] used exclusively qualitative methods of analysis to assess 18F-FDG uptake/accumulation within the walls of affected vessels in GCA/PMR patients (Figure 2).

Three of these articles [25, 26, 36] proposed a visual grading scale exclusively based on vascular $18 \mathrm{~F}-\mathrm{FDG}$ uptake and 5 studies [27, 31, 32, 38, 39] used a visual grading score based on the vessel-to-liver ratio. The remaining 2 studies $[28,30]$ defined each examination as positive or negative (i.e., abnormal versus normal), without specifying a positive threshold (Table 2).

In his first prospective study, Blockmans et al. [25] proposed a visual 4-point scale with scores ranging from 0 to 3, which was described as follows: a 0 score indicated no visualization of blood vessels; a score of 1 meant minimal $18 \mathrm{~F}$ FDG uptake, a score of 2 an increased 18F-FDG uptake, and a score of $318 \mathrm{~F}-F D G$ a pronounced uptake. Blood vessels of the lower and upper limbs and the thoracic arteries were individually defined as positive for inflammatory involvement if the score was $\geq 2$.

Brodmann et al. [30] examined 22 consecutive patients with clinical diagnosis of GCA confirmed by DUS: $18 \mathrm{~F}-$ FDG PET scans were rated as negative or positive, without a definition of the criterion used (Figure 3). Meller et al. [27] proposed a visual grading scale, used by four other studies [31, 32, 38, 39], where large vessel 18F-FDG uptake was compared with that of the liver. According to this method, zero was defined as no uptake, 1 as uptake present but lower than liver uptake, 2 as uptake similar to liver uptake, and 3 as uptake higher than liver uptake (Figure 4). Three [27, 32, 39] out of these 5 studies concluded that a grade $\geq 2$ for the thoracic aorta and a grade $\geq 1$ in the other vascular regions were positive criteria for vasculitis. A smooth linear or long segmental pattern of 18F-FDG uptake in the aorta and its main branches, with an intensity higher than the liver uptake, was regarded as findings highly suggestive for GCA.

\subsection{Semiquantitative Methods of Assessment of 18F-FDG PET.} Six studies [21, 24, 33, 35, 40, 41] used a semiquantitative scoring method to evaluate vascular uptake (Table 3 ). Blockmans, in two prospective studies [21, 33], proposed a semiquantitative system that evaluates 18F-FDG uptake in 7 different vascular regions (thoracic aorta, abdominal aorta, subclavian arteries, axillary arteries, carotid arteries, iliac arteries, and femoral arteries) with the following grading: 0: no uptake; 1: minimal but not negligible uptake; 2: clearly increased uptake; and 3: very marked uptake. Based on this first evaluation, a total vascular score (TVS) was calculated, ranging from 0 (no vascular FDG uptake in any of the 7 vascular regions) to 21 (maximum vascular FDG uptake in 


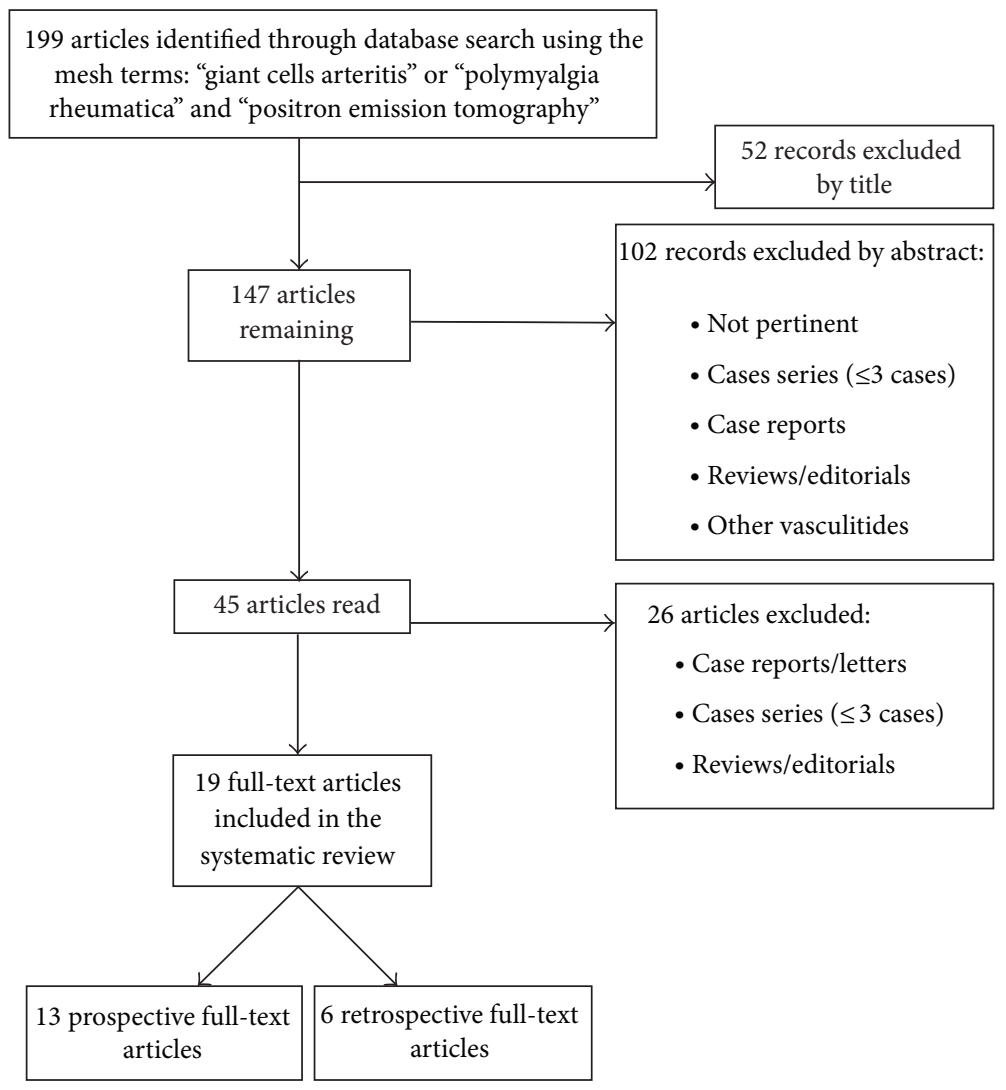

FIGURE 1: Flowchart of the review process.

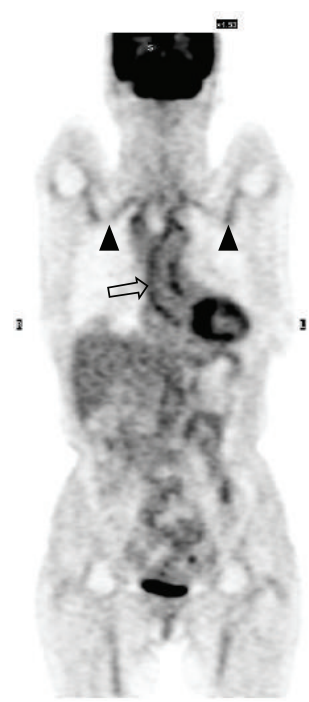

(a)

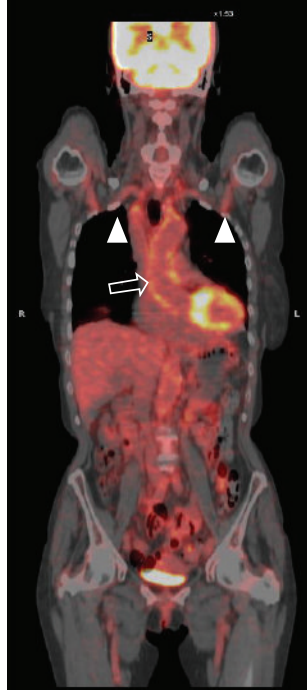

(b)

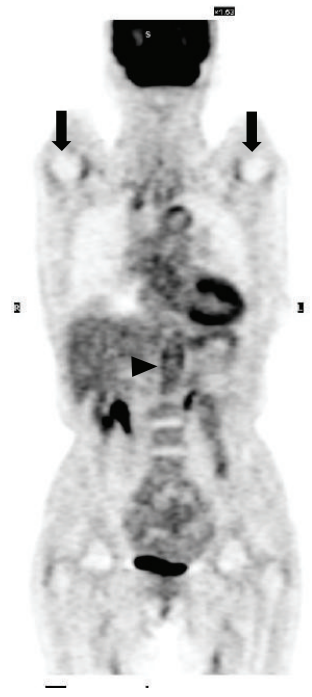

(c)

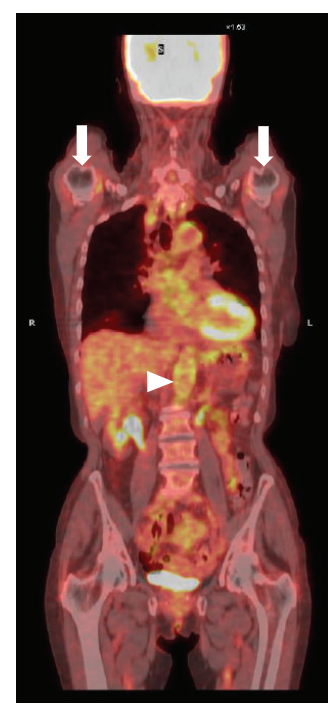

(d)

FIGURE 2: 65-year-old female patient with 18F FDG PET-CT findings indicating the clinical association of polymyalgia rheumatica and giant cell arteritis. Coronal PET (a) and PET-CT (b) images demonstrate a significant tracer uptake of the walls of the ascending aorta, aortic arch (void arrows in (a) and (b)), and subclavian arteries (arrowheads in (a) and (b)). The second pair of coronal PET (c) and PET-CT (d) images demonstrate the inflammatory involvement of the abdominal aorta (arrowheads in (c) and (d)). A bilateral uptake of the tracer of the glenohumeral joints is also seen (solid arrows). 


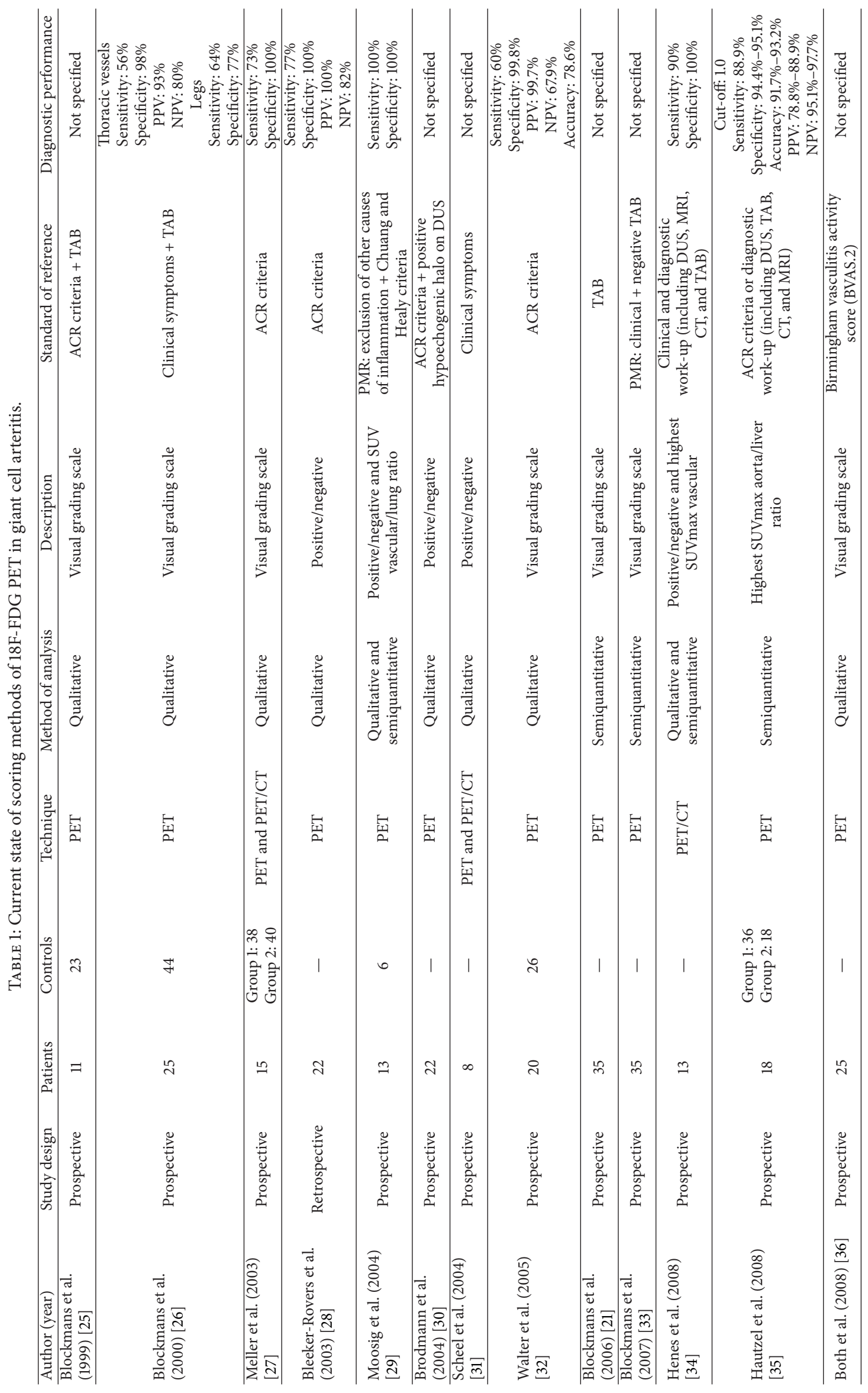




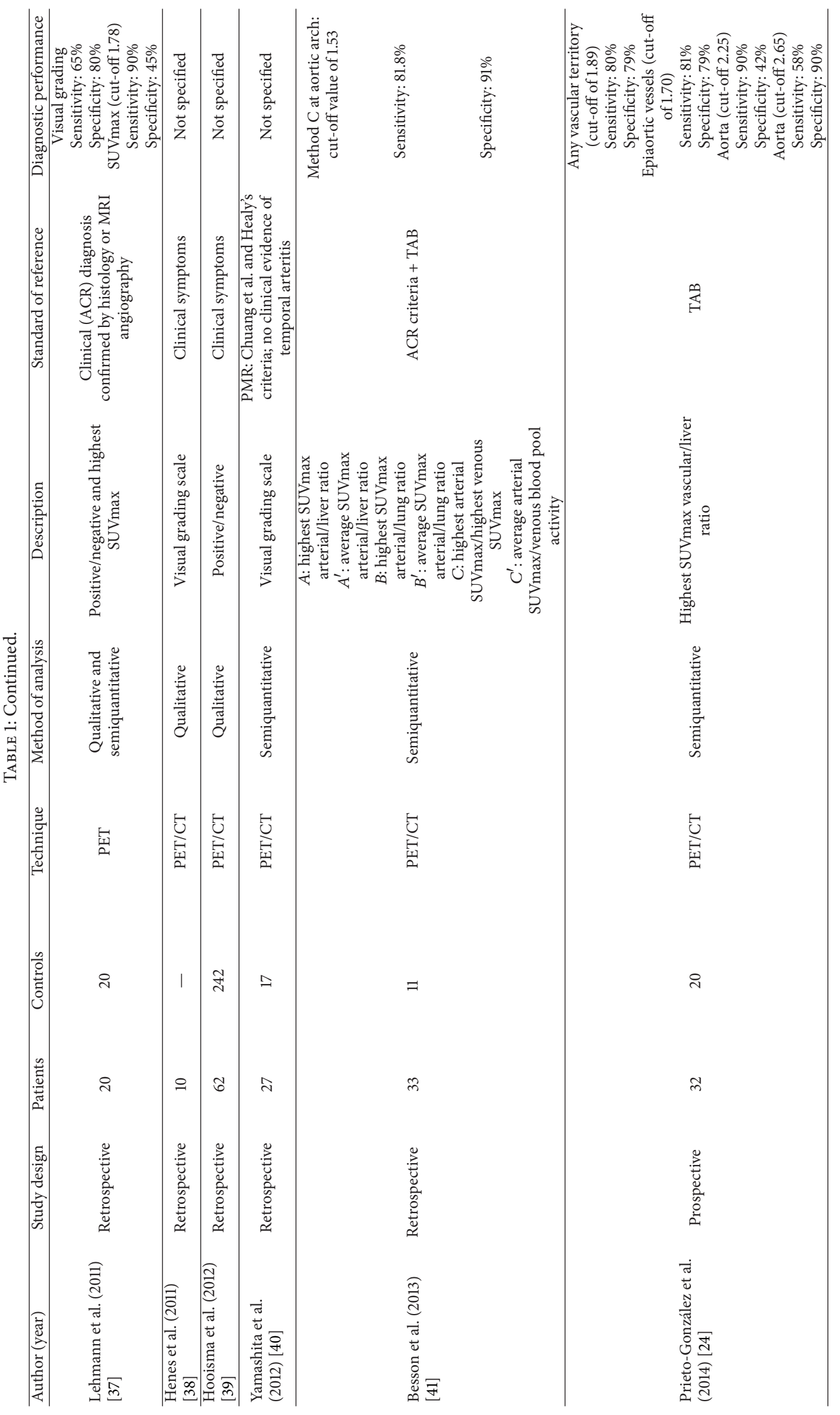




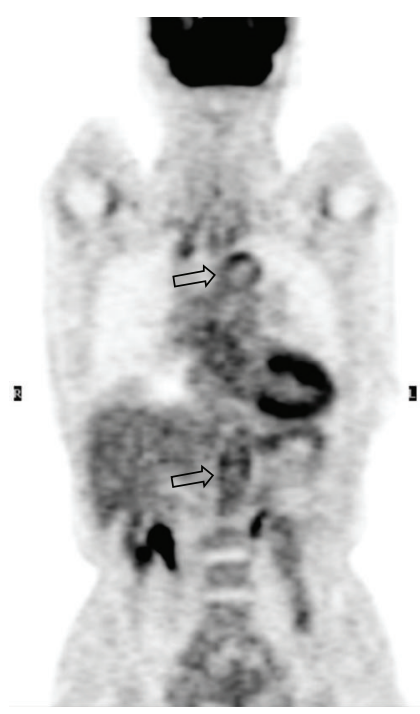

(a)

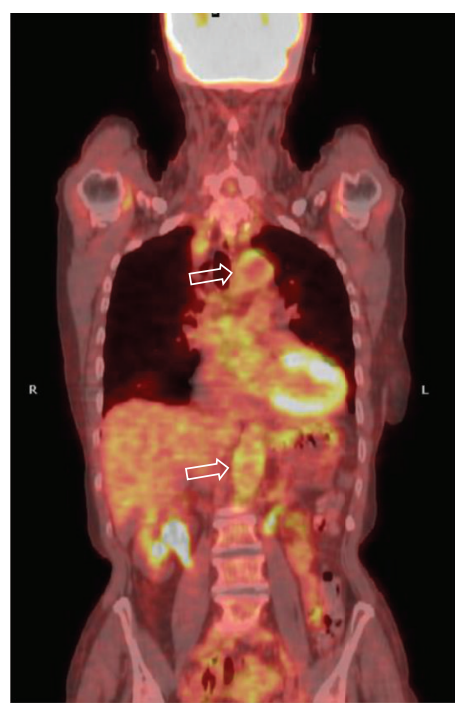

(b)

FIgURE 3: 77-year-old female patient with clinical and PET-CT findings of giant cell arteritis. Coronal PET (a) and PET-CT (b) scans demonstrate the inflammatory involvement of aortic arch and abdominal aorta (void arrows), which is clearly appreciable by means of an immediate qualitative assessment of the images.

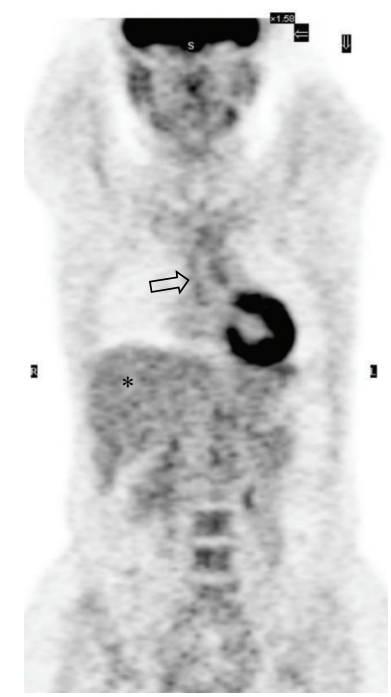

(a)

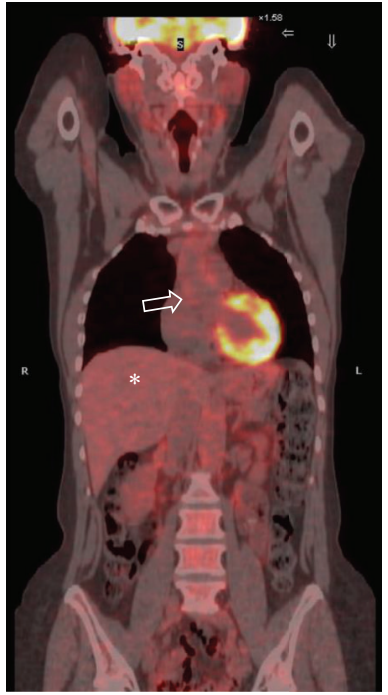

(b)

FIGURE 4: 80-year-old male patient with clinical and PET-CT findings of giant cell arteritis. In this patient, coronal PET (a) and PET-CT (b) images demonstrate 18F FDG uptake of the walls of the ascending thoracic aorta (void arrows). The tracer uptake is similar to that of the liver parenchyma (asterisk), corresponding to grade 2 (significant vascular inflammation) according to the visual grading score proposed by Meller et al.

TABLE 2: 18F-FDG PET and PET/CT qualitative diagnostic criteria.

\begin{tabular}{ll}
\hline Number of studies & Description \\
\hline 2 & Positive/negative or normal/abnormal \\
\hline 3 & Visual grading scale \\
\hline 5 & $0:$ none; 1: slight; 2: marked; 3: intense \\
\hline
\end{tabular}




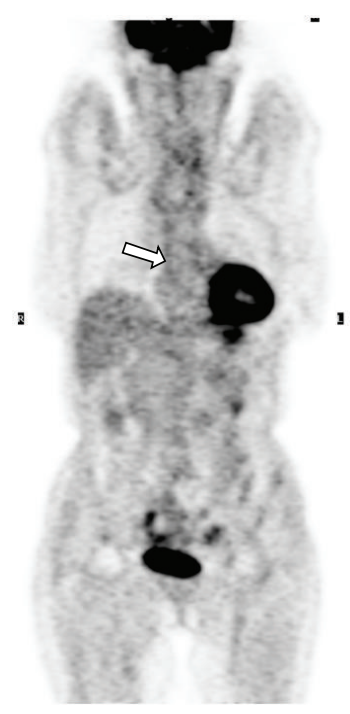

(a)

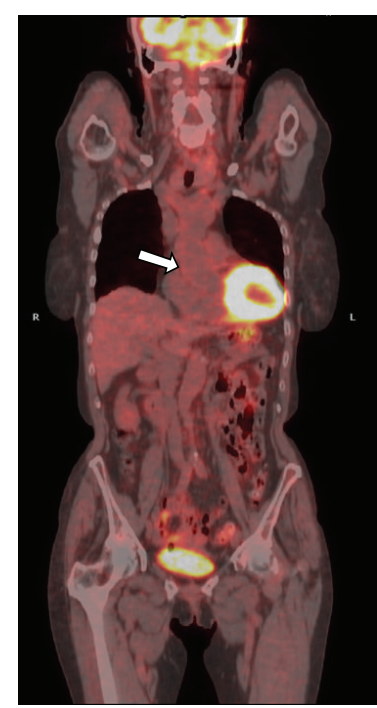

(b)

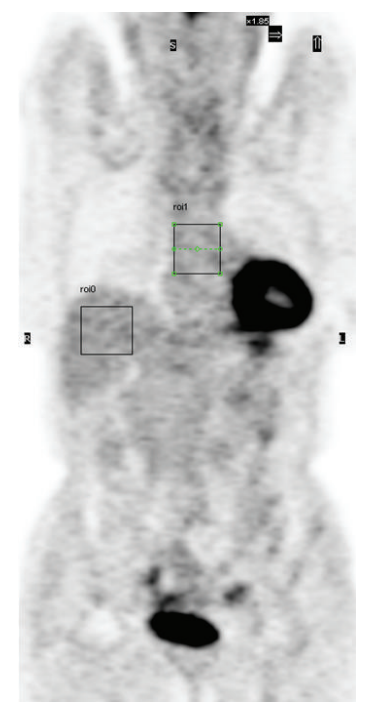

(c)

FIGURE 5: 65-year-old female patient with polymyalgia rheumatica and suspected giant cell arteritis. An immediate qualitative visual assessment of the coronal PET-CT scan ((a) and (b)) led to the diagnosis of inflammatory involvement of the ascending thoracic aorta (white arrows). In this patient, the semiquantitative method of analysis proposed by Hautzel et al. was further applied (aorta-to-liver SUVmax ratio). Placing a ROI on the ascending thoracic aorta in the coronal PET image (c), a SUVmax of 1.6 was obtained. The SUVmax obtained by drawing the same ROI comprehensive on the liver (c) was 2.2, and the resulting aorta-to-liver SUVmax ratio was 0.7, below the cut-off value of 1 for diagnosing significant vascular inflammation. This is an example of discrepancy between qualitative and semiquantitative methods of image analysis.

TABle 3: 18F-FDG PET and PET/CT semiquantitative diagnostic criteria.

\begin{tabular}{ll}
\hline Number of studies & Description \\
\hline 3 & Visual grading scale (0-3) and TVS \\
3 & Highest SUVmax vascular/liver ratio \\
1 & Average SUVmax vascular/liver ratio \\
1 & Highest SUVmax vascular/lung ratio \\
1 & Average SUVmax vascular/lung ratio \\
1 & Highest SUVmax arterial/venous ratio \\
1 & Average SUVmax arterial/venous ratio \\
\hline
\end{tabular}

all 7 locations). They found a mean TVS of $6 \pm 0.2$ at the time of diagnosis in 29 of 35 patients with GCA, with or without PMR. They also found a significantly lower mean TVS of $0.8 \pm 1.7$ in $31 \%$ of isolated PMR cases.

Hautzel et al. [35] introduced a semiquantitative aorta-toliver SUVmax ratio. In their prospective study they found an optimal relationship of sensitivity to specificity at an aortato-liver ratio of 1.0 , even in patients with altered hepatic metabolism (Figure 5).

In their retrospective controlled study [41], Besson et al. compared three different semiquantitative approaches. The first method was derived from that described by Hautzel et al. [35], including two variants for normalizing the arterial activity to the liver uptake (i.e., highest SUVmax vascular/liver ratio and average SUVmax vascular/liver ratio) [35, 41]. The second approach was adapted from that described by Moosig et al. [29] and included two variants, where the arterial activity was normalized to the lung uptake (i.e., highest SUVmax vascular/lung ratio and average SUVmax vascular/lung ratio). The third approach had not been previously applied in GCA/PMR, but it was originally tested in atherosclerosis patients to evaluate arterial wall inflammation [42]. It was based on the arterial-to-blood pool uptake ratio and included two variants: in the first, the highest arterial SUVmax was normalized to the highest venous SUVmax; in the second, the average arterial SUVmax was normalized to the venous blood pool activity. The latter one was calculated by averaging the values obtained from eight ROIs drawn on axial 18F-FDG images in the right internal jugular vein.

3.3. Combined Qualitative and Semiquantitative Methods. Three studies $[29,34,37]$ used a first qualitative analysis to diagnose or rule out the presence of vasculitis, performing a further semiquantitative assessment on vascular 18F-FDG uptake.

In the study of Moosig et al. [29], the visual examination of PET scans showed an increased tracer uptake in the aorta or in its major branches in 12 out of 13 included patients. For the semiquantitative assessment, 9 vascular areas were identified and sampled by placing different regions of interest. A peripheral region of the lung served to represent the background uptake, and vessel-to-lung SUVmax ratio was calculated. According to this method, Moosig et al. found that patients with active disease had significantly greater $18 \mathrm{~F}-\mathrm{FDG}$ uptake than control patients (mean ROI index of 1.58 versus 0.93). 
Henes et al. [34] focused their analysis on the vessel showing the highest accumulation of the tracer at a first qualitative visual assessment. Further, they measured the maximum SUV on 6 locations of the selected vascular region. They found a mean SUVmax of 3.4 for all patients. The SUVmax was 3.9 in untreated patients versus 3.0 in patients under medical treatment.

Lehmann et al. [37] used two different diagnostic approaches for image assessment: a first visual analysis of vessel wall uptake compared to the background activity of liver, followed by a semiquantitative reevaluation, consisting of calculating the SUVmax in predefined regions of interest. They found that the SUVmax cut-off value of 1.78 is characterized by a high sensitivity (90\% versus $65 \%$ of the qualitative visual assessment) and a low specificity (45\% versus $80 \%$ of the qualitative visual assessment).

\section{Discussion}

The aim of our systematic review, which included a total of 442 cases of GCA patients, with or without PMR symptoms, and 535 controls, was to analyze the different qualitative and semiquantitative methods for assessing the presence and grading the severity of GCA-related vascular inflammation on $18 \mathrm{~F}-\mathrm{FDG}$ PET scans that have been proposed in the literature. We found the need for a standardized 18F-FDG PET interpretation in order to optimize the diagnostic performance of this imaging technique. Indeed, the lack of a standardized reading approach to defining vascular inflammation remains a critical issue and may lead to misclassification. Currently, the diagnosis of GCA is mainly based on clinical evaluation, laboratory findings, and temporal artery biopsy (TAB). The American College of Rheumatology diagnostic criteria for GCA do not include any imaging modality [43].

In patients with a distinctive clinical presentation, the diagnosis of GCA is not difficult and TAB is able to confirm the clinical suspicion. By contrast, a correct diagnosis may become challenging when symptoms are nonspecific, given the wide range of clinical manifestations of GCA $[3,5,44]$. Currently, TAB is still the diagnostic standard of reference $[1,2]$, but its routine clinical application is hampered by low sensitivity with a high false-negative rate (15\%-40\%) [4549] and the concrete risk of underdetection [1, 9-12, 50]. Furthermore, the involvement of the thoracic aorta or its main branches, which is present in more than $45 \%$ of newly diagnosed patients with GCA, is associated with a negative TAB in $50 \%$ of reported cases $[20,51-54]$. 18 F-FDG PET is a noninvasive, whole-body technique that is able to detect vascular involvement in GCA patients, with or without PMR symptoms $[25,26]$. Other imaging techniques (i.e., DUS, Doppler ultrasonography, CT, and MRI) have been proposed for the assessment of vascular inflammation in GCA patients. However, while they are able to demonstrate anatomical changes in the affected vessels (mural thickening, dilatation and aneurysms, and enhancement of perivascular connective tissue) if the inflammatory process is well established, they are not sensitive enough to diagnose early inflammatory changes which are potentially reversible [28]. Furthermore, patient follow-up and the assessment of response to medical treatment are not easy to perform on the basis of morphological information alone [27, 28].

DUS of temporal arteries has emerged as a useful alternative tool when temporal biopsy cannot be performed [52], but this technique is not able to demonstrate the involvement of thoracic vessels. 18F-FDG may be particularly helpful in patients with inconclusive TAB and/or DUS results $[55,56]$.

From the results of our review, we found that qualitative analysis of $18 \mathrm{~F}-\mathrm{FDG}$ uptake is the most widely adopted method for assessing the presence and grading the activity of GCA-related vascular inflammation on 18F-FDG PET scans (13 out of 19 original papers). Qualitative analysis has been used both to perform dichotomous assessment (i.e., confirm or rule out the presence of vascular inflammation) [28-31, 34, $35,37,39]$ and to grade the severity of vascular involvement according to ordinal scales, with the 18F-FDG uptake of the vessel wall being visually analyzed or compared with that of a reference structure [25-27, 32, 34, 36]. Among visual grading systems, the vessel-to-liver ratio is the most frequently used. With regard to diagnostic performance, a previous systematic meta-analysis compared different qualitative and semiquantitative methods for assessing vasculitis on 18F-FDG PET, reporting a pooled sensitivity and a specificity of $80 \%$ and $89 \%$, respectively [51].

The strength of qualitative methods, in addition to being more immediate and less time-consuming than semiquantitative ones, lies in their high specificity and small number of false positives, while sensitivity ranges from $56 \%$ to $77 \%$. Moreover, qualitative methods display high interobserver agreement and intraobserver reproducibility ( $90 \%$ and 93.3\%, resp.) [32]. By contrast, most authors [24, 26, 28, 41, $42,57-60]$ have reported that when qualitative methods are applied, the diagnostic discrimination between vasculitis and atherosclerosis may be a critical issue.

However, there is a general consensus that $18 \mathrm{~F}-\mathrm{FDG}$ vascular uptake in vasculitis should be higher than in atherosclerosis. For these reasons, mild vascular 18F-FDG uptake, lower than or equal to that of the liver (i.e., grades 1 and 2 according to scoring systems that use the liver as a reference structure), is not indicative of GCA inflammatory involvement. The site of tracer accumulation and its changes in response to medical treatment have also been considered to distinguish large vessel vasculitis from atherosclerosis [60].

A complementary contrast-enhanced CT acquired immediately after 18F-FDG PET/CT may provide additional information on the morphology of the affected vessels and the presence of calcifications within the arterial wall [34]. The metabolic information obtained from 18F-FDG PET, combined with the demonstration of wall enhancement and thickness assessed by contrast-enhanced CT, could constitute an effective approach to the evaluation of extracranial GCA, thereby enabling a better differentiation between GCA- and atherosclerosis-related 18F-FDG vascular uptake.

With regard to the semiquantitative methods, some authors have proposed the evaluation of arterial SUVmax $[24,34,37]$, while others have normalized arterial SUVmax 
to the background activity, represented by the mean uptake value of a selected reference structure [29, 35, 41].

On comparing a SUVmax-based approach without normalization with visual qualitative analysis, Lehmann et al. [37] found high sensitivity (90\% versus 65\%) and low specificity ( $45 \%$ versus $80 \%$ ) when a cut-off value of 1.78 was used. By contrast, Besson et al. [41] and Prieto-González et al. [24] found that SUVmax cut-off values obtained without normalization were population-specific and could not be applied to the general population.

From the results of our analysis, we observed that semiquantitative methods normalized to the background activity seem to outperform qualitative approaches and semiquantitative methods without normalization. We also noticed that the power of discrimination between GCA and control groups also depends on the anatomical structure chosen to represent the background uptake.

Besson et al. showed some limitations in the use of the liver as the background for normalization in GCA [41]. In particular, they found that the liver SUVmax differed significantly between patients and controls and that the liver SUVmax values in the GCA group were significantly higher than in the control group. Indeed, systemic inflammation affects liver metabolism and can influence the calculation of liver uptake values.

According to the results of our study, an interesting semiquantitative approach is the arterial-to-background ratio proposed by Moosig et al. [29]. These authors adopted a two-step procedure for assessing their patients. Initially, they applied a dichotomous qualitative method in order to identify positive cases; they then adopted a semiquantitative method, normalizing vascular $18 \mathrm{~F}-\mathrm{FDG}$ uptake to the lung. Indeed, the background activity of the lung displays low inter- and intrapatient variability owing to the low physiological uptake of $18 \mathrm{~F}-\mathrm{FDG}$. Using this two-step procedure, Moosig et al. obtained maximal diagnostic performance values (i.e., both sensitivity and specificity of $100 \%$ ) higher than those yielded by both qualitative and semiquantitative methods. Besson et al. [41] normalized vascular 18F-FDG uptake to the lung by means of a semiquantitative method similar to that of Moosig et al. and confirmed that the lung SUVmax values did not differ significantly between patients and controls. On the other hand, without performing the two-step combined procedure of Moosig et al. [29], Besson et al. [41] found lower values of sensitivity and specificity ( 81.8 and $72.7 \%$, resp.).

Among all the semiquantitative methods for the assessment of 18F-FDG PET in GCA, the aortic-to-blood pool uptake ratio seems to outperform the other methods when liver and lung are used as reference structures for determining the background uptake.

This approach, tested by Rudd et al. in atherosclerosis to evaluate arterial wall inflammation, has proved to be robust and highly reproducible [57]. Rudd et al. normalized the arterial SUVmax values to blood pool activity, represented by the mean of 8 ROIs drawn in venous vessels (i.e., the inferior vena cava or the internal jugular vein). Besson et al. adapted this method in order to assess PET examinations of 11 GCA patients and 11 controls matched for age and sex and found high diagnostic sensitivity (81.8\%) and specificity (91\%).

\section{Conclusion}

In conclusion, $18 \mathrm{~F}-\mathrm{FDG}$ PET has been shown to have an important role in the diagnosis of extracranial vascular involvement in patients with GCA/PMR.

Qualitative methods are more specific than semiquantitative ones, but they have lower sensitivity. The aorticto-blood pool uptake ratio is a promising semiquantitative method of analysis for the detection and grading of arterial inflammation. The normalization of the arterial wall uptake to the background activity of venous blood pool provides a good reference to assess vascular inflammation. Further prospective studies involving larger cohorts of GCA/PMR patients are required to better define the role of aortic-toblood pool ratio as a reference method for the assessment of vasculitis in GCA patients.

\section{Conflict of Interests}

The authors declare that there is no conflict of interests regarding the publication of this paper.

\section{References}

[1] C. Salvarani, F. Cantini, L. Boiardi, and G. G. Hunder, "Polymyalgia rheumatica and giant-cell arteritis," New England Journal of Medicine, vol. 347, no. 4, pp. 261-271, 2002.

[2] M. A. Gonzalez-Gay, C. Garcia-Porrua, J. A. Miranda-Filloy, and J. Martin, "Giant cell arteritis and polymyalgia rheumatica: pathophysiology and management," Drugs and Aging, vol. 23, no. 8, pp. 627-649, 2006.

[3] D. M. Nuenninghoff, G. G. Hunder, T. J. H. Christianson, R. L. McClelland, and E. L. Matteson, "Incidence and predictors of large-artery complication (aortic aneurysm, aortic dissection, and/or large-artery stenosis) in patients with giant cell arteritis: a population-based study over 50 years," Arthritis and Rheumatism, vol. 48, no. 12, pp. 3522-3531, 2003.

[4] J. M. Evans, W. M. O’Fallon, and G. G. Hunder, "Increased incidence of aortic aneurysm and dissection in giant cell (temporal) arteritis: a population-based study," Annals of Internal Medicine, vol. 122, no. 7, pp. 502-507, 1995.

[5] T. Bongartz and E. L. Matteson, "Large-vessel involvement in giant cell arteritis," Current Opinion in Rheumatology, vol. 18, no. 1, pp. 10-17, 2006.

[6] S. Barber, "Myalgic syndrome with constitutional effects," Annals of the Rheumatic Diseases, vol. 16, pp. 230-237, 1957.

[7] J. M. Evans and G. G. Hunder, "Giant cell arteritis, temporal arteritis, and polymyalgia rheumatica in a Danish county. A prospective investigation, 1982-1985," Arthritis and Rheumatism, vol. 30, no. 3, pp. 294-299, 1987.

[8] C. Schaufelberger, B.-A. Bengtsson, and R. Andersson, "Epidemiology and mortality in 220 patients with polymyalgia rheumatica," British Journal of Rheumatology, vol. 34, no. 3, pp. 261-264, 1995.

[9] P. Elling, A. T. Olsson, and H. Elling, "Synchronous variations of the incidence of temporal arteritis and polymyalgia rheumatica in different regions of Denmark; association with epidemics of Mycoplasma pneumoniae infection," Journal of Rheumatology, vol. 23, no. 1, pp. 112-119, 1996. 
[10] F. Cantini, L. Niccoli, L. Storri et al., "Are polymyalgia rheumatic and giant cell arteritis the same disease?" Seminars in Arthritis and Rheumatism, vol. 33, no. 5, pp. 294-301, 2004.

[11] C. Salvarani, C. S. Crowson, W. M. O’Fallon, G. G. Hunder, and S. E. Gabriel, "Reappraisal of the epidemiology of giant cell arteritis in Olmsted County, Minnesota, over a fifty-year period," Arthritis Care and Research, vol. 51, no. 2, pp. 264-268, 2004.

[12] E. Nordborg and C. Nordborg, "Giant cell arteritis: epidemiological clues to its pathogenesis and an update on its treatment," Rheumatology, vol. 42, no. 3, pp. 413-421, 2003.

[13] J. T. Gran and G. Myklebust, "The incidence of polymyalgia rheumatica and temporal arteritis in the county of Aust Agder, South Norway: a prospective study 1987-94," The Journal of Rheumatology, vol. 24, no. 9, pp. 1739-1743, 1997.

[14] P. Franzen, S. Sutinen, and J. von Knorring, "Giant cell arteritis and polymyalgia rheumatica in a region of Finland: an epidemiologic, clinical and pathologic study, 1984-1988," Journal of Rheumatology, vol. 19, no. 2, pp. 273-280, 1992.

[15] C. Salvarani, L. Boiardi, V. Mantovani et al., "HLA-DRB1 alleles associated with polymyalgia rheumatica in northern Italy: correlation with disease severity," Annals of the Rheumatic Diseases, vol. 58, no. 5, pp. 303-308, 1999.

[16] M. A. González-Gay, M. M. Amoli, C. Garcia-Porrua, and W. E. R. Ollier, "Genetic markers of disease susceptibility and severity in giant cell arteritis and polymyalgia rheumatica," Seminars in Arthritis and Rheumatism, vol. 33, no. 1, pp. 38-48, 2003.

[17] M. Bas-Lando, G. S. Breuer, Y. Berkun, M. Mates, M. Sonnenblick, and G. Nesher, "The incidence of giant cell arteritis in Jerusalem over a 25-year period: annual and seasonal fluctuations," Clinical and Experimental Rheumatology, vol. 25, supplement 44, no. 1, pp. S15-S17, 2007.

[18] P. Duhaut, S. Bosshard, and J.-P. Ducroix, "Is giant cell arteritis an infectious disease? Biological and epidemiological evidence," Presse Medicale, vol. 33, no. 19, pp. 1403-1408, 2004.

[19] C. Salvarani, F. Cantini, and G. G. Hunder, "Polymyalgia rheumatica and giant-cell arteritis," The Lancet, vol. 372, no. 9634, pp. 234-245, 2008.

[20] M. A. Gonzalez-Gay, C. Garcia-Porrua, A. Piñeiro, R. PegoReigosa, J. Llorca, and G. G. Hunder, "Aortic aneurysm and dissection in patients with biopsy-proven giant cell arteritis from Northwestern Spain: a population-based study," Medicine, vol. 83, no. 6, pp. 335-341, 2004.

[21] D. Blockmans, L. de Ceuninck, S. Vanderschueren, D. Knockaert, L. Mortelmans, and H. Bobbaers, "Repetitive 18Ffluorodeoxyglucose positron emission tomography in giant cell arteritis: a prospective study of 35 patients," Arthritis Care and Research, vol. 55, no. 1, pp. 131-137, 2006.

[22] S. Prieto-González, P. Arguis, A. García-Martínez et al., "Large vessel involvement in biopsy-proven giant cell arteritis: prospective study in 40 newly diagnosed patients using CT angiography," Annals of the Rheumatic Diseases, vol. 71, no. 7, pp. 1170-1176, 2012.

[23] M. Aschwanden, F. Kesten, M. Stern et al., "Vascular involvement in patients with giant cell arteritis determined by duplex sonography of 2x11 arterial regions," Annals of the Rheumatic Diseases, vol. 69, no. 7, pp. 1356-1359, 2010.

[24] S. Prieto-González, M. Depetris, A. García-Martínez et al., "Positron emission tomography assessment of large vessel inflammation in patients with newly diagnosed, biopsy-proven giant cell arteritis: a prospective, case-control study," Annals of the Rheumatic Diseases, vol. 73, no. 7, pp. 1388-1392, 2014.
[25] D. Blockmans, A. Maes, S. Stroobants et al., "New arguments for a vasculitic nature of polymyalgia rheumatica using positron emission tomography," Rheumatology, vol. 38, no. 5, pp. 444447, 1999.

[26] D. Blockmans, S. Stroobants, A. Maes, and L. Mortelmans, "Positron emission tomography in giant cell arteritis and polymyalgia rheumatica: evidence for inflammation of the aortic arch," The American Journal of Medicine, vol. 108, no. 3, pp. 246-249, 2000.

[27] J. Meller, F. Strutz, U. Siefker et al., "Early diagnosis and followup of aortitis with $\left[{ }^{18} \mathrm{~F}\right] \mathrm{FDG}$ PET and MRI," European Journal of Nuclear Medicine and Molecular Imaging, vol. 30, no. 5, pp. 730-736, 2003.

[28] C. P. Bleeker-Rovers, S. J. H. Bredie, J. W. M. van der Meer, F. H. M. Corstens, and W. J. G. Oyen, "F-I8-fluorodeoxyglucose positron emmission tomography in diagnosis and follow-up of patients with different types of vasculitis," Netherlands Journal of Medicine, vol. 61, no. 10, pp. 323-329, 2003.

[29] F. Moosig, N. Czech, C. Mehl et al., "Correlation between 18fluorodeoxyglucose accumulation in large vessels and serological markers of inflammation in polymyalgia rheumatica: a quantitative PET study," Annals of the Rheumatic Diseases, vol. 63, no. 7, pp. 870-873, 2004.

[30] M. Brodmann, R. W. Lipp, A. Passath, G. Seinost, E. Pabst, and E. Pilger, "The role of 2-18F-fluoro-2-deoxy-D-glucose positron emission tomography in the diagnosis of giant cell arteritis of the temporal arteries," Rheumatology, vol. 43, no. 2, pp. 241-242, 2004.

[31] A. K. Scheel, J. Meller, R. Vosshenrich et al., "Diagnosis and follow up of aortitis in the elderly," Annals of the Rheumatic Diseases, vol. 63, no. 11, pp. 1507-1510, 2004.

[32] M. A. Walter, R. A. Melzer, C. Schindler, J. Müller-Brand, A. Tyndall, and E. U. Nitzsche, "The value of [18F]FDG-PET in the diagnosis of large-vessel vasculitis and the assessment of activity and extent of disease," European Journal of Nuclear Medicine and Molecular Imaging, vol. 32, no. 6, pp. 674-681, 2005.

[33] D. Blockmans, L. de Ceuninck, S. Vanderschueren, D. Knockaert, L. Mortelmans, and H. Bobbaers, "Repetitive 18fluorodeoxyglucose positron emission tomography in isolated polymyalgia rheumatica: a prospective study in 35 patients," Rheumatology, vol. 46, no. 4, pp. 672-677, 2007.

[34] J. C. Henes, M. Müller, J. Krieger et al., “[18F] FDG-PET/CT as a new and sensitive imaging method for the diagnosis of largevessel vasculitis," Clinical and Experimental Rheumatology, vol. 26, supplement 49, no. 3, pp. S47-S52, 2008.

[35] H. Hautzel, O. Sander, A. Heinzel, M. Schneider, and H. Müller, "Assessment of large-vessel involvement in giant cell arteritis with 18F-FDG PET: introducing an ROC-analysis-based cutoff ratio," Journal of Nuclear Medicine, vol. 49, no. 7, pp. 1107-1113, 2008.

[36] M. Both, K. Ahmadi-Simab, M. Reuter et al., "MRI and FDGPET in the assessment of inflammatory aortic arch syndrome in complicated courses of giant cell arteritis," Annals of the Rheumatic Diseases, vol. 67, no. 7, pp. 1030-1033, 2008.

[37] P. Lehmann, S. Buchtala, N. Achajew et al., "18F-FDG PET as a diagnostic procedure in large vessel vasculitis-a controlled, blinded re-examination of routine PET scans," Clinical Rheumatology, vol. 30, no. 1, pp. 37-42, 2011.

[38] J. C. Henes, M. Mueller, C. Pfannenberg, L. Kanz, and I. Koetter, "Cyclophosphamide for large vessel vasculitis: assessment of response by PET/CT," Clinical and Experimental Rheumatology, vol. 29, no. 1, supplement 64, pp. S43-S48, 2011. 
[39] G. A. Hooisma, H. Balink, P. M. Houtman, R. H. J. A. Slart, and K. D. F. Lensen, "Parameters related to a positive test result for FDG PET(/CT) for large vessel vasculitis: a multicenter retrospective study," Clinical Rheumatology, vol. 31, no. 5, pp. 861-871, 2012.

[40] H. Yamashita, K. Kubota, Y. Takahashi et al., "Whole-body fluorodeoxyglucose positron emission tomography/ computed tomography in patients with active polymyalgia rheumatica: evidence for distinctive bursitis and large-vessel vasculitis," Modern Rheumatology, vol. 22, no. 5, pp. 705-711, 2012.

[41] F. L. Besson, H. de Boysson, J. Parienti, G. Bouvard, B. Bienvenu, and D. Agostini, "Towards an optimal semiquantitative approach in giant cell arteritis: an ${ }^{18}$ F-FDG PET/CT case-control study," European Journal of Nuclear Medicine and Molecular Imaging, vol. 41, no. 1, pp. 155-166, 2013.

[42] M. Tatsumi, C. Cohade, Y. Nakamoto, and R. L. Wahl, "Fluorodeoxyglucose uptake in the aortic wall at PET/CT: possible finding for active atherosclerosis," Radiology, vol. 229, no. 3, pp. 831-837, 2003.

[43] G. G. Hunder, D. A. Bloch, B. A. Michel et al., "The American College of Rheumatology 1990 criteria for the classification of giant cell arteritis," Arthritis and Rheumatism, vol. 33, no. 8, pp. 1122-1128, 1990.

[44] D. M. Nuenninghoff, G. G. Hunder, T. J. H. Christianson, R. L. McClelland, and E. L. Matteson, "Incidence and predictors of aortic aneurysm, aortic dissection and large artery stenosis in patients with giant cell arteritis in Olmsted county, MN, over a 50- year period," Arthritis \& Rheumatology, vol. 46, supplement 9, p. S183, 2002.

[45] E. W. T. Chong and A. J. Robertson, "Is temporal artery biopsy a worthwhile procedure?" ANZ Journal of Surgery, vol. 75, no. 6, pp. 388-391, 2005.

[46] R. Taylor-Gjevre, M. Vo, D. Shukla, and L. Resch, “Temporal artery biopsy for giant cell arteritis," Journal of Rheumatology, vol. 32, no. 7, pp. 1279-1282, 2005.

[47] A. M. Roth, L. Milsow, and J. L. Keltner, "The ultimate diagnoses of patients undergoing temporal artery biopsies," Archives of Ophthalmology, vol. 102, no. 6, pp. 901-903, 1984.

[48] M. A. Gonzalez-Gay, C. Garcia-Porrua, J. Llorca, C. GonzalezLouzao, and P. Rodriguez-Ledo, "Biopsy-negative giant cell arteritis: clinical spectrum and predictive factors for positive temporal artery biopsy," Seminars in Arthritis and Rheumatism, vol. 30, no. 4, pp. 249-256, 2001.

[49] S. Hall, S. Persellin, J. T. Lie, P. C. O'Brien, L. T. Kurland, and G. G. Hunder, "The therapeutic impact of temporal artery biopsy," The Lancet, vol. 2, no. 8361, pp. 1217-1220, 1983.

[50] R. C. Brooks and S. R. McGee, "Diagnostic dilemmas in polymyalgia rheumatica," Archives of Internal Medicine, vol. 157, no. 2, pp. 162-168, 1997.

[51] F. L. Besson, J. Parienti, B. Bienvenu et al., "Diagnostic performance of 18F-fluorodeoxyglucose positron emission tomography in giant cell arteritis: a systematic review and metaanalysis," European Journal of Nuclear Medicine and Molecular Imaging, vol. 38, no. 9, pp. 1764-1772, 2011.

[52] W. A. Schmidt, A. Seifert, E. Gromnica-ihle, A. Krause, and A. Natusch, "Ultrasound of proximal upper extremity arteries to increase the diagnostic yield in large-vessel giant cell arteritis," Rheumatology, vol. 47, no. 1, pp. 96-101, 2008.

[53] C. Agard, J. Barrier, B. Dupas et al., "Aortic involvement in recent-onset giant cell (temporal) arteritis: a case-control prospective study using helical aortic computed tomodensitometric scan," Arthritis Care and Research, vol. 59, no. 5, pp. 670676, 2008.

[54] A. Brack, V. Martinez-Taboada, A. Stanson, J. J. Goronzy, and C. M. Weyand, "Disease pattern in cranial and large-vessel giant cell arteritis," Arthritis \& Rheumatology, vol. 42, pp. 311-317, 1999.

[55] M. A. Cimmino, D. Camellino, F. Paparo et al., "High frequency of capsular knee involvement in polymyalgia rheumatica /giant cell arteritis patients studied by positron emission tomography," Rheumatology, vol. 52, no. 10, pp. 1865-1872, 2013.

[56] F. Jamar, J. Buscombe, A. Chiti et al., "EANM/SNMMI guideline for ${ }^{18}$ F-FDG use in inflammation and infection," Journal of Nuclear Medicine, vol. 54, no. 4, pp. 647-658, 2013.

[57] J. H. F. Rudd, K. S. Myers, S. Bansilal et al., "Atherosclerosis inflammation imaging with ${ }^{18}$ F-FDG PET: carotid, iliac, and femoral uptake reproducibility, quantification methods, and recommendations," Journal of Nuclear Medicine, vol. 49, no. 6, pp. 871-878, 2008.

[58] M. P. S. Dunphy, A. Freiman, S. M. Larson, and H. W. Strauss, "Association of vascular 18F-FDG uptake with vascular calcification," Journal of Nuclear Medicine, vol. 46, no. 8, pp. 1278-1284, 2005.

[59] M. Yun, S. Jang, A. Cucchiara, A. B. Newberg, and A. Alavi, "18F FDG uptake in the large arteries: a correlation study with the atherogenic risk factors," Seminars in Nuclear Medicine, vol. 32, no. 1, pp. 70-76, 2002.

[60] T. Belhocine, D. Blockmans, R. Hustinx, J. Vandevivere, and L. Mortelmans, "Imaging of large vessel vasculitis with 18FDG PET: illusion or reality? A critical review of the literature data," European Journal of Nuclear Medicine and Molecular Imaging, vol. 30, no. 9, pp. 1305-1313, 2003. 


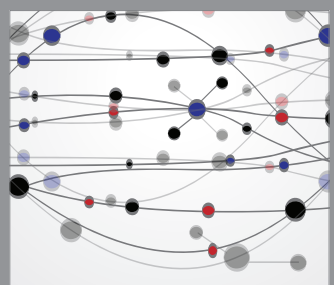

The Scientific World Journal
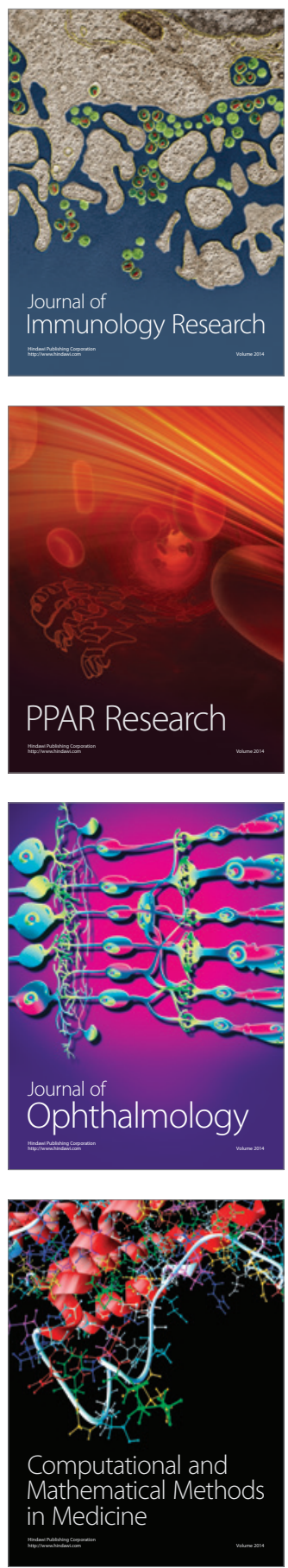

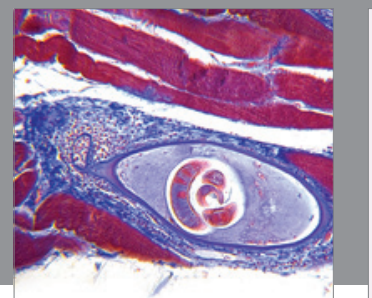

Gastroenterology

Research and Practice
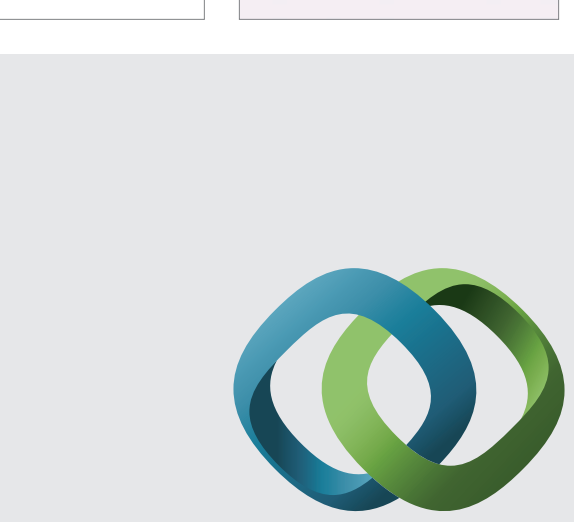

\section{Hindawi}

Submit your manuscripts at

http://www.hindawi.com
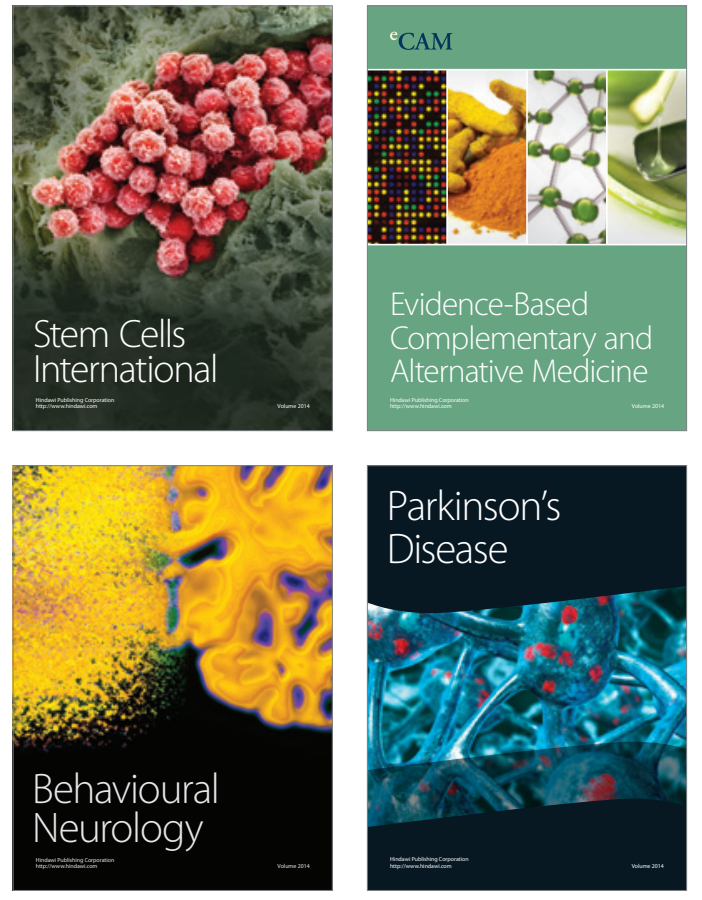
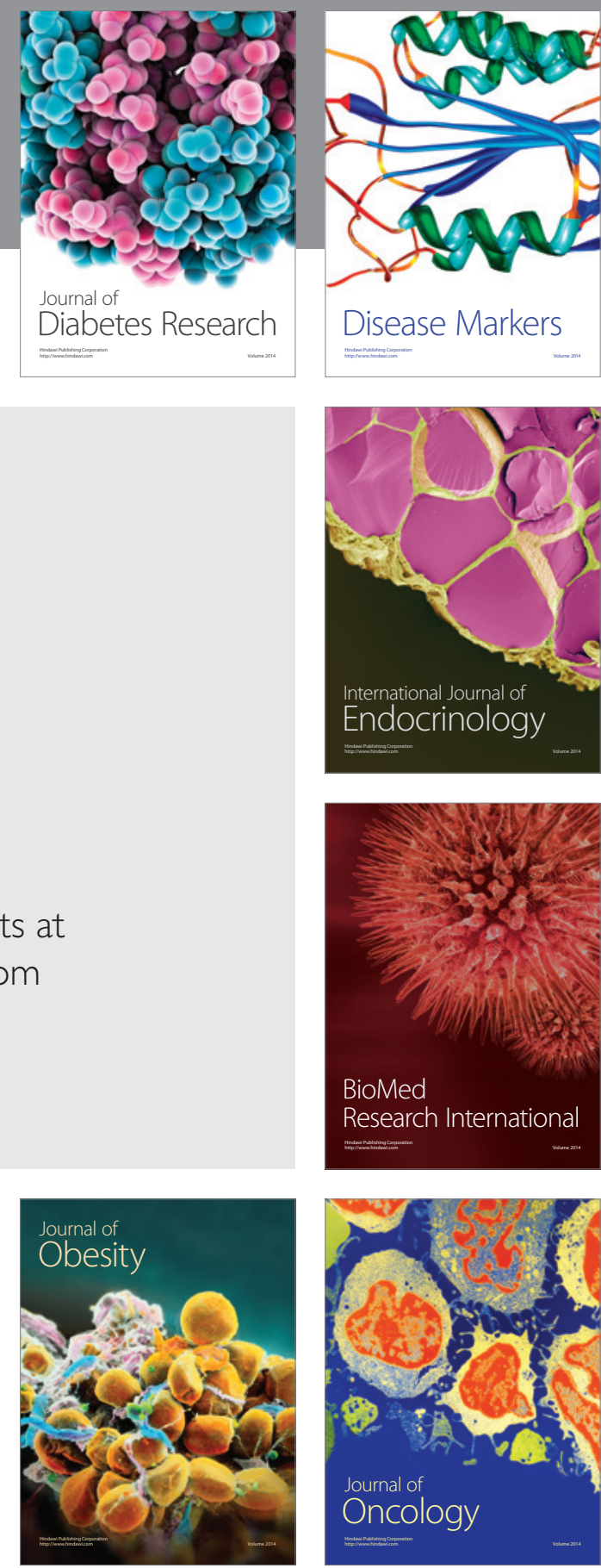

Disease Markers
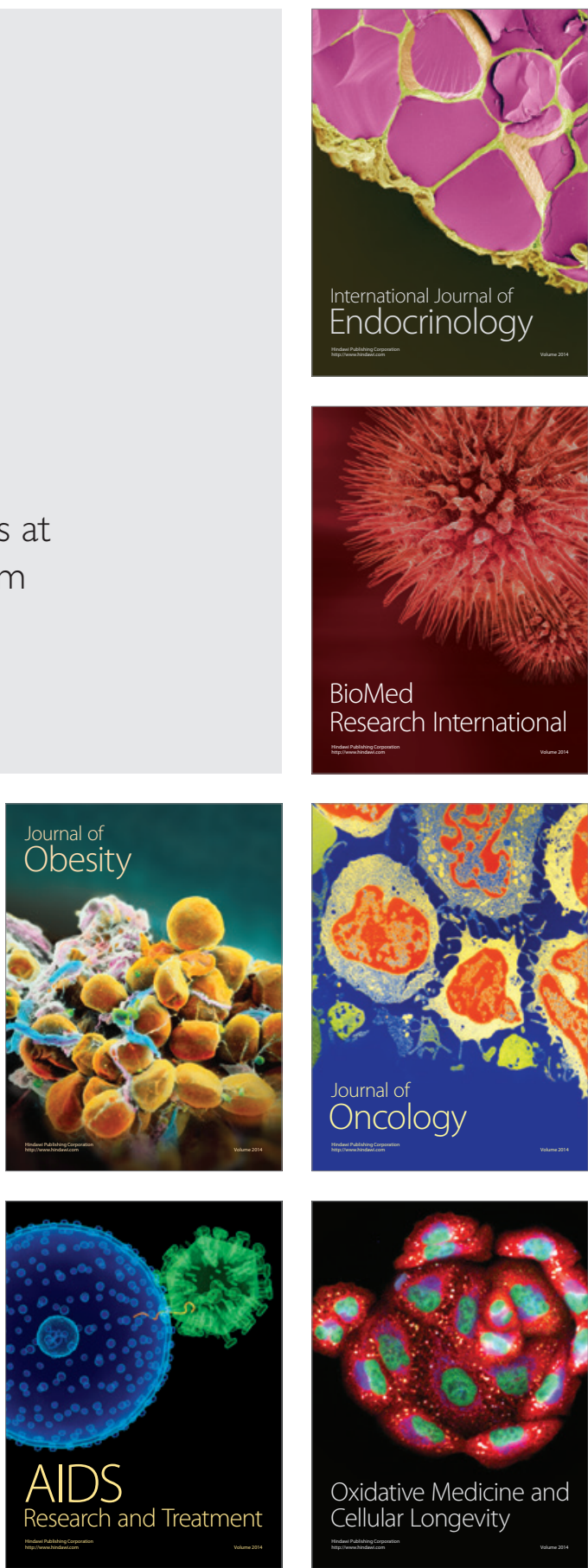